\title{
Silicone oil-intraocular lens interaction: which lens to use?
}

\author{
E McLoone, G Mahon, D Archer, R Best
}

\begin{abstract}
Aim-To determine a suitable intraocular lens for implantation in patients at high risk of lens exposure to silicone oil in their lifetime.

Methods-PMMA, AcrySof, AR40, AQUA-Sense, and Raysoft lenses were examined. Each lens was immersed for 5 minute intervals in balanced salt solution (BSS), in stained silicone oil, and again in BSS before being photographed in air and in BSS. Percentage silicone oil coverage of the lens optic was determined.

Results-The mean percentage coating (MPC) for the lens biomaterials ranged from $5.2 \%$ to $21.5 \%$. The Raysoft lens had significantly less oil coverage when statistically compared with the other lens types $(\mathrm{p}<0.001)$.

Conclusion-A Raysoft (Rayner) lens is a suitable lens for implantation in patients who are at risk of severe vitreoretinal disease.

(Br F Ophthalmol 2001;85:543-545)
\end{abstract}

Silicone oil is increasingly used as an adjunct in the management of the more complex vitreoretinal disorders ${ }^{12}$ such as giant retinal tears, severe proliferative vitreoretinopathy, or cytomegalovirus (CMV) related retinal detachments. However, since 1994 several case studies $^{3-5}$ have described an interaction between silicone oil and silicone intraocular lenses (IOLs) which, reportedly, causes significant visual loss and aberration for the patient as well as obstruction of the vitreoretinal surgeon's intraoperative view. This complication has the potential to occur either when silicone oil is employed as an intravitreal tamponade in retinal surgery in a pseudophakic patient or when combined silicone oil removal and cataract surgery, with lens implantation, is undertaken. ${ }^{67}$

In order to determine which lens type would

Department of Ophthalmology, Queen's University of Belfast, Institute of Clinical Science, Grosvenor Road, Belfast BT12 6BA, UK E McLoone

G Mahon

D Archer

R Best

Correspondence to: Professor D Archer eibhlin-mcloone@hotmail.com

Accepted for publication 31 October 2000 tions) lenses. IOLs $(n=6)$ of each type were immersed in balanced salt solution (BSS) at $37^{\circ} \mathrm{C}$ for 5 minutes to simulate an aqueous environment. The lenses were then immersed in 1000 centistoke silicone oil (Adato Sil 1000). The silicone oil was initially dyed by adding $40 \mathrm{mg} / \mathrm{ml}$ of Sudan IV, a lipid soluble histological stain. ${ }^{8}$ The excess dye particles were removed by centrifugation at $13500 \mathrm{rpm}$ for 5 minutes (Micro-centaur-MSE, UK). This colouring enabled better visualisation of the silicone oil droplets on photography. After 5 minutes the lenses were removed from the oil. Senn $e t a l^{9}$ have shown that the length of exposure to silicone oil has no bearing on the oil-lens interaction. The lenses were finally reimmersed in BSS for a further 5 minutes. Each lens was examined under the microscope and gross photographs were obtained both in air and in BSS.

The percentage silicone oil coating on the front surface of each lens optic was determined by image analysis using UTHSCSA Image Tool software. ${ }^{10}$ All results were statistically analysed using a one way analysis of variance test, a multiple comparison test (Student-NewmanKeuls) and the arcsin transformation. ${ }^{11}$

\section{Results}

The silicone oil formed a uniform coating on the lens optics in the dry state and, as such, was difficult to visualise. However, after immersion in BSS, oil droplets formed. Figures 1A and B illustrate this difference in silicone oil coverage of a PMMA IOL in air and in BSS. As submersion in BSS was more representative of the physiological state of the eye, analysis was performed on these results.

Figures 1B-F demonstrate the gross photographic appearances of silicone oil coverage on the lens biomaterials. The MPC of oil on PMMA (Fig 1B), AcrySof (Fig 1C), AR40 (Fig 1D), and AQUA-Sense (Fig 1E) lenses was $20.8 \%, 17.1 \%, 21.5 \%$, and $17.8 \%$ respectively. Raysoft lenses (Fig 1F) had the least silicone oil adherence with a MPC of $5.2 \%$. Results are summarised in Table 1.

Initial data analysis, using a one way analysis of variance (ANOVA) test, indicated a high degree of reproducibility of the results as well as a significant difference in silicone oil coverage between the groups of lens biomaterials $(\mathrm{p}<0.001)$. A multiple comparison method (Student-Newman-Keuls test) showed that the Raysoft intraocular lens had significantly less silicone oil coverage compared with the other lens types. No significant differences in oil coverage were noted on comparison of the other lenses. As the percentage oil coverage of the optics within each lens group was not normally distributed, the data were transformed using the arcsin transformation. Analysis of the transformed data confirmed the above results. 

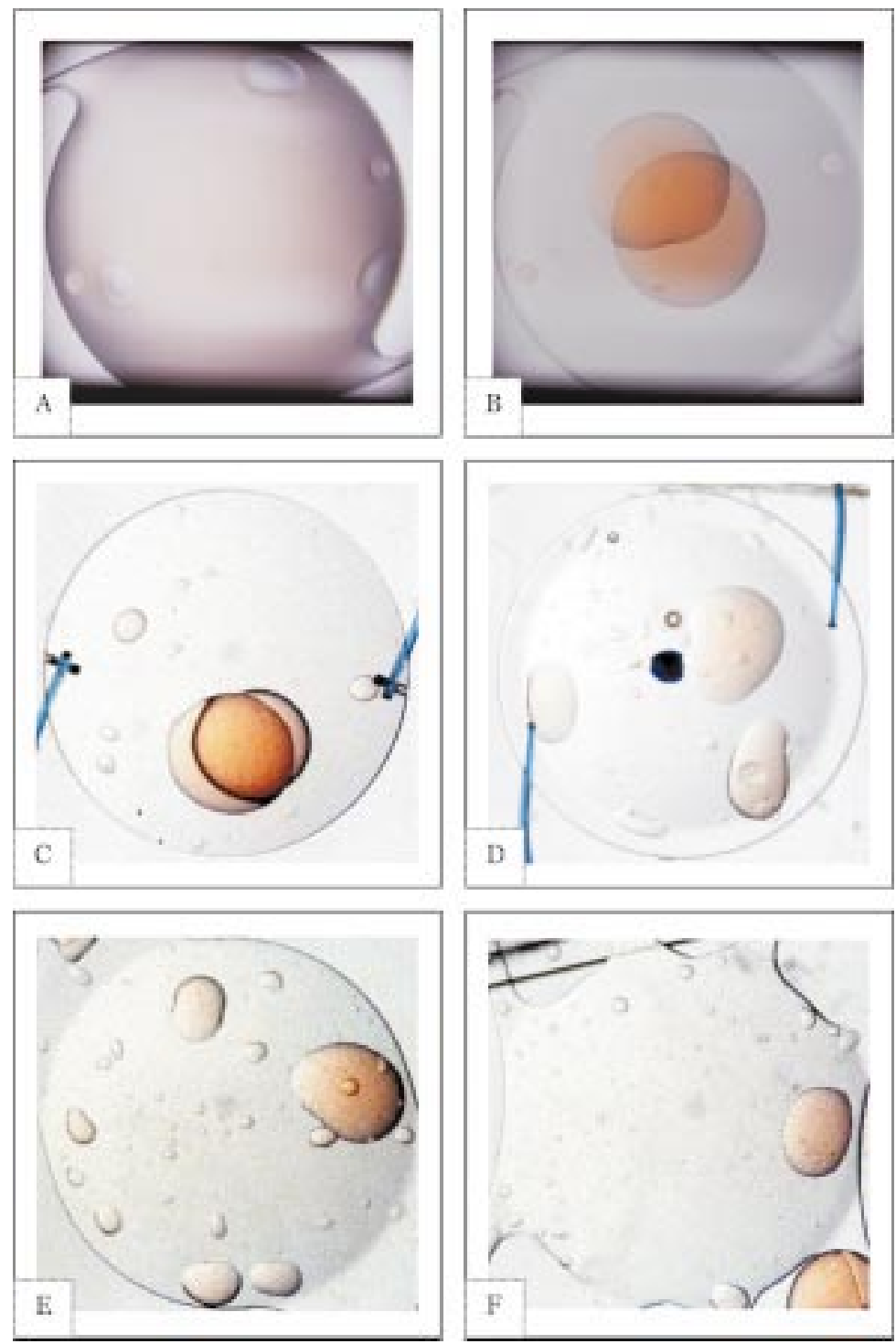

Figure 1 Gross photographs of intraocular lens biomaterials with adherent silicone oil. $(A)$ PMMA (Ophthalmic Innovations) lens in air (the dry state). (B) PMMA lens in BSS (the aqueous state). Mean percentage coating 20.8\%. (C) AcrySof (Alcon) lens. Mean percentage coating $17.1 \%$. (D) AR40 (Allergan) lens. Mean percentage coating $21.5 \%$. (E) AQUA-Sense (Ophthalmic Innovations) lens. Mean percentage coating $17.8 \%$. $(F)$ Raysoft (Rayner) lens. Mean percentage coating 5.2\%

Table 1 Lens chemical structure and mean percentage silicone oil adhesion

\begin{tabular}{|c|c|c|c|c|}
\hline Lens type & Polymer composition & $M P C(\%)$ & Range (\%) & $S D(\%)$ \\
\hline PMMA & Methylmethacrylate & 20.8 & $18.9-24.7$ & 2.3 \\
\hline AcrySof & $\begin{array}{l}\text { Phenylethylacrylate } \\
\text { Phenylethylmethacrylate }\end{array}$ & 17.1 & $13.9-21.8$ & 3.2 \\
\hline AR40 & $\begin{array}{l}\text { Ethylacrylate } \\
\text { Ethylmethacrylate } \\
\text { Trifluoroethylmethacrylate }\end{array}$ & 21.5 & $17.2-24.9$ & 2.7 \\
\hline AQUA- Sense & $\begin{array}{l}\text { Hydroxyethylmethacrylate } \\
\text { Ethoxyethylmethacrylate }\end{array}$ & 17.8 & $13.2-26.9$ & 4.9 \\
\hline Raysoft & Hydroxyethylmethacrylate & 5.2 & $3.0-7.5$ & 1.7 \\
\hline
\end{tabular}

\section{Discussion}

It is now well established that silicone oil can interact with various intraocular lens biomaterials with a potential for reducing the optical quality of the lens. ${ }^{512}$ Apple et $a l^{13}$ published the results of an in vitro experiment comparing the degree of adherence of silicone oil (5000 centistokes) to various rigid and foldable IOL designs. Interaction of silicone oil was maximal with silicone lenses (Allergan Medical Optics,
Staar Surgical, and Chiron Vision). In all cases the mean percentage coating was $100 \%$. PMMA IOLs (Pharmacia-Upjohn) had a MPC of $20.7 \%$. Soft acrylic IOLs (Alcon and Allergan Medical Optics) had a MPC of $33.7 \%$.

An in vivo study published by Khawly et $a l^{14}$ supported the finding of significant adherence of silicone oil (1000 centistokes) on all of 10 silicone IOLs (Allergan Medical Optics) examined. However, adherent silicone oil remained on only two of nine PMMA IOLs (Alcon) and on none of 10 acrylic IOLs (Alcon).

In our experimental study, PMMA lenses (Ophthalmic Innovations) had a mean percentage silicone oil coating of $20.8 \%$. This correlates well with Apple et al's finding of a MPC of $20.7 \%$ on PMMA lenses (PharmaciaUpjohn). Apple et al looked at both AcrySof and AR40 soft acrylic lenses as a single lens biomaterial and reported a MPC of $33.7 \%$. We found a lower MPC of oil on these lenses when examined as separate groups. The MPC on AcrySof (Alcon) and AR40 (Allergan) lenses was $17.1 \%$ and $21.5 \%$ respectively.

No statistical difference in the degree of silicone oil adherence to the optics of PMMA, AcrySof, AR40, or AQUA-Sense lenses was found in our study. However, the Raysoft intraocular lens had significantly less silicone oil coverage compared with the other lens types examined.

The silicone oil used in this study was 1000 centistokes (Adato Sil 1000). In other departments, 5000 centistokes silicone oil may be in use. Senn et $a l^{9}$ have demonstrated no obvious differences between the two viscosities of silicone oil in terms of oil-lens interaction.

The underlying mechanism for the interaction between silicone oil and intraocular lens biomaterials has yet to be determined.

All intraocular lenses, with the exception of silicone lenses, are composed of an acrylate/ methacrylate backbone. ${ }^{15}$ The side chains give the various biomaterials their unique properties. The molecular structure of each lens, as given in Table 1, and its environment determines the polar forces and dispersive (Van der Waals) forces ${ }^{16}$ involved in the interaction.

Apple et $a l^{13}$ have postulated that it is the hydrophobia of silicone oil which influences its interaction with intraocular lenses. The more hydrophobic a lens biomaterial is the more the adherence of silicone oil; the more hydrophilic, the less the adherence.

The physical parameters of hydrophobicity and hydrophilicity are determined using the sessile drop method of contact angle measurements. However, Cunanan et $a l^{17}$ have demonstrated that all their study lens biomaterials were in fact hydrophobic by the sessile drop contact angle method. However, measurements by the captive bubble method in a hydrated environment resulted in lower contact angles with differences emerging between the lens types. The air bubble contact angles for PMMA, AR40, and AcrySof lenses were $66.2 \%, 64.0 \%$, and $45.9 \%$ respectively indicating that the AcrySof lens was more hydrophilic than the other two lens types. Therefore, it is 
not surprising that the AcrySof lens had the least silicone oil adhesion of these lenses. Raysoft and AQUA-Sense lenses were not analysed by Cunanan et al and therefore contact angle measurements in a hydrated environment are not yet available for comparison. Perhaps, the AQUA-Sense hydrogel lens is relatively hydrophobic on captive bubble testing thus accounting for its increased silicone oil coating compared with the Rayner hydrogel lens.

In conclusion, our experimental data showed that the Raysoft (Rayner) IOL, with a mean percentage oil coating of $5.2 \%$, had the least silicone oil adherence of the various lens biomaterials examined. Statistical analysis indicated that this result was significant $(p<0.001)$. Therefore, the Raysoft intraocular lens is a suitable lens for implantation in patients at risk of severe vitreoretinal disease. However, our study was an in vitro experiment looking at the effect of 1000 centistoke silicone oil on intraocular lens biomaterials. Conclusions must therefore be drawn with some caution. More extensive in vivo studies examining the effects of both 1000 and 5000 centistoke silicone oil on acrylic lenses are necessary to confirm our results.

1 Scott JD. Silicone oil as an instrument. In: Ryan SJ, ed. Retina. 2nd ed. St Louis: CV Mosby 1994; vol 3, chapte 135:2181-9.

2 The Silicone Study Group. Vitrectomy with silicone oil or perfluoropropane gas in eyes with severe proliferative vitreoretinopathy: results of a randomised clinical trial. Silicone Study report 2. Arch Ophthalmol 1992;110:780-92.
3 Kusaka S, Kodama T, Ohashi Y. Condensation of silicone oil on the posterior surface of a silicone intraocular lens during vitrectomy. Am f Ophthalmol 1996;121:574-5.

4 Bartz-Schmidt KU, Konen W, Esser P, et al. Intraocular silicone lenses and silicone oil. Klin Monatsbl Augenheilkd $1995 ; 207 ; 162-6$.

5 Apple DJ, Federman JL, Krolicki TJ, et al. Irreversible silicone oil adhesion to silicone intraocular lenses. Ophthalmology 1996;103:1555-61.

6 Batterbury M, Wong D, Williams R, et al. The adherence of silicone oil to standard and heparin-coated PMMA intraocular lenses. Eye 1994;8:547-9.

7 Dick B, Stoffelns B, Pavlovic S, et al. Interaction of silicone oil with various intraocular lenses. A light and scanning electron microscopy study. Klin Monatsbl Augenheilkd 1997;211;192-206.

8 Langefeld S, Kirchhof B, Meinert H, et al. A new way of removing silicone oil from the surface of silicone intraocular lenses. Graefes Arch Clin Exp Ophthalmol 1999;237:201-

9 Senn P, Schmid MK, Schipper I, et al. Interaction between silicone oil and silicone intraocular lenses: an in vitro study. Ophthalmic Surg Lasers 1997;28:776-9.

10 UTHSCSA ImageTool software developed at the University of Texas Health Science Center at San Antonio, Texas and available from the Internet by anonymous FTP from maxrad6.uthscsa.edu.

11 Snedecor GW, Cochran WG. Statistical methods. 6th ed. Iowa State: University Press, 327.

12 Stolba U, Binder S, Velikay $\mathrm{M}$, et al. Intraocular silicone lenses in silicone oil: an experimental study. Graefes Arch Clin Exp Ophthalmol 1996;234:55-7.

13 Apple DJ, Isaacs RT, Kent DG, et al. Silicone oil adhesion to intraocular lenses: an experimental study comparing various biomaterials. $\mathcal{F}$ Cataract Refract Surg 1997;23:53644.

14 Khawly JA, Lambert RJ, Jaffe GJ. Intraocular lens changes after short- and long-term exposure to intraocular silicone oil: an in vivo study. Ophthalmology 1998;105:1227-33.

15 Chehade M, Elder MJ. Intraocular lens materials and styles: a review. Aust NZ F Ophthalmol 1997;25:255-63.

16 Andrade JD. Interfacial phenomena and biomaterials. Med Instrum 1973;7:110-20.

17 Cunanan CM, Ghazizadeh M, Buchen SY, et al. Contactangle analysis of intraocular lenses. 7 Cataract Refract Surg $1998 ; 24: 341-51$. 